\title{
Risk Factors for Endometrial Cancer among Post-menopausal Women in South Africa
}

\author{
Suddhasattwa Ray ${ }^{1}$, Shadi Zohorinia ${ }^{2}$, Dipankar Bhattacharyya ${ }^{1}$, Sayan \\ Chakravorty ${ }^{1}$, Sonia .S. Ray ${ }^{3}$
}

${ }^{1}$ Pinnacle Cancer Centres Bangalore, India. ${ }^{2}$ Baran Radiation Oncology Centre, Khorramabad, Iran. ${ }^{3}$ Lincoln American University, George town, Guyana.

\begin{abstract}
Background: National Cancer Registry of South Africa reported the highest incidence of uterine cancer in Gauteng Proviance. Among uterine cancer patients reported in the Department of Radiation Oncology, Johannesburg Hospital, more than $85 \%$ are suffering in endometrial cancers. Here, in this study we tried to explore the relation between factors causing increased blood oestrogen levels, like, contraceptive pills, early menarche and delayed menopause (increases the length of menstruation), nulliparity and overweight by more than $25 \mathrm{~kg}$ and endometrial cancer among black female population of Greater Johannesburg. In this present study, we also studied how non-sex hormone related factors like hypertension, diabetes, family history, obesity and smoking habits are affecting the occurrence of endometrial cancer in Greater Johannesburg females. Methods: This study is based on post-menopausal female black population of Johannesburg who were physically examined with the help of transvaginal sonar. Their oestrogen level in serum was checked. Their family history, menarche and menopause age, pregnancy and others were collected through questionnaires. Results: The mean age of menarche and menopause was found to be 13.80 and 53.80 years respectively. Hypertension, elevated serum oestradiol level, nulliparity, obesity and diabetes are significantly high among study group. Moreover our study suggests no such relation between smoking habit and endometrial cancer. Conclusion: Continued exposure of endometrium to oestrogen increases the chance of endometrial cancer. The length of menstruation time and nulliparity appeared as vital factors for endometrial cancer. Moreover, adipocytes secreting oestrogen and diabetes are also related to the onset of endometrial cancer among post-menopausal female population.
\end{abstract}

Keywords: Endometrial cancer- oestrogen- overweight- smoking- post-menopausal female population

Asian Pac J Cancer Biol, 4 (2), 41-45

\section{Introduction}

Uterine cancer is the fourth most frequent cancer in women. Each year, 142,000 women develop endometrial cancer worldwide and an estimated 42,000 women die from this cancer. Tumors of the uterine fundus comprise the most common group of gynecologic malignancies. Approximately $97 \%$ of tumors arise within the epithelium of the uterine lining and are categorized as endometrial carcinomas; the remaining 3 percent of uterine cancers are sarcomas. As per the National Cancer Registry of 1993-95, uterine cancer is the fourth commonest cancer in the black females of South Africa with an incidence of $3.7 \%$ of cancers. In the African female population, uterine
Submission Date: 05/18/2019 Acceptance Date: 07/23/2019

cancer has the highest incidence in Gauteng Province (within South Africa).

The normal endometrium is a hormonally responsive tissue. Oestrogenic stimulation produces cellular growth and glandular proliferation, which is cyclically balanced by the maturational effects of progesterone. Abnormal proliferation and neoplastic transformation of the endometrium have been associated with chronic unopposed exposure to oestrogenic stimulation [1-2]. These include oral intake of exogenous oestrogen (without progestins), oestrogen-secreting tumours, low parity, extended periods of anovulation, early menarche and late menopause [3]. Since menarche and menopause are commonly associated with absent or irregular ovulation, women

\footnotetext{
Corresponding Author:

Dr. Suddhaswatta Ray

Pinnacle Cancer Centres Bangalore, India.

Email: Drssray2002@yahoo.co.uk
} 
who experience early onset or late cessation of ovarian function are more likely to have additional oestrogenic exposure. Moreover shifting of hormonal balance towards progesterone during pregnancy interrupts the prolonged oestrogen exposure thereby reducing the risk of endometrial cancer whereas nulliparity increases chance of getting endometrium cancer by increasing the span of oestrogenic exposure [4-5].

Morbidly obese women also have a greater risk of endometrial cancer, presumably because their adipocytes are able to convert androstenedione of adrenal origin to estrone, a weak circulating oestrogen [6]. Postmenopausal women has high levels of androstenedione and testosterone which are converted to oestrogens. Further exogenous administration of oestrogen during oestrogen Replacement Therapy (ERT) for treating postmenopausal symptoms, usage of tamoxifen for treating breast cancer and oral contraceptive pills (oestrogen alone) for birth control are associated with increased incidence of endometrial cancer. Women with polycystic ovaries have high oestrogen levels and low levels of progestins than normal thus increases a woman's chance of getting endometrial cancer.

This study focused on the assessment of direct link of unopposed oestrogen with age of menarche and menopause, menstruation time in years, parity, obesity, Body Mass Index (BMI), and the use of oral contraceptive pills with the aid of a questionnaire. This study also investigated the association of non sex hormone related aetiological factors such as hypertension, diabetes, family history of colorectal /endometrial malignancy and smoking with the incidence of endometrial cancer.

\section{Materials and Methods}

\section{Patients}

Thirty black female patients of age above 55 years with histologically proven carcinoma of the endometrium were included this study with their informed consent. The control group consisted of thirty age matched African women from Soweto, Gauteng, having no signs and symptoms of malignancy for last 5 years and were not on hormone replacement therapy.

\section{Physical Examination}

Transvaginal sonar was performed 3 times on these groups. Endometrial thickness of less than $4 \mathrm{~mm}$ was considered as the cut off value for normal [7-8]. Apart from this, blood pressure, height, body weight, body mass index (BMI) were also estimated.

\section{Assessment of serum oestradiol level}

Blood samples were collected from both patient and control groups to check serum oestradiol level. Blood samples ( $5 \mathrm{ml}$ of clotted sample) for oestradiol level estimation by immunoassay were collected from 30 patients having endometrial cancer before initiation of any treatment and from 30 normal subjects. Samples were allowed to clot before centrifugation and then serum was collected. The ADVIA Centaur Oestradiol -6 Ш assay is a competitive immunoassay using direct chemiluminescent technology. Oestradiol in the patient sample competes with acridinium ester- labelled oestradiol for a limited amount of rabbit anti oestradiol antibody. Rabbit anti - oestradiol is captured by mouse anti - rabbit $\mathrm{IgG}$, which is coupled to paramagnetic particles in the solid phase. An inverse relationship exists between the amount of oestradiol present in the patient sample and the amount of relative lights units detected by the system. Blood oestradiol levels were analysed in the Department of Chemical Pathology, Johannesburg Hospital, South Africa.

\section{Patient History}

Patient history was collected by direct enquiry using a questionnaire consisted of questions on nulliparity, smoking habits, use of oral contraceptive pills, family/ personal history of endometrial and colorectal malignancy, duration of menstruation period in years, age of menarche, age of menopause etc.

\section{Statistical analysis}

For the t-test, using a significance level of 0.05 and with a power of 0.9 , a minimum of 30 subjects in each group were taken. Data analysis was done using SAS software. ANOVA Test was used to evaluate the blood oestradiol levels, age at menopause, menstruation time in years and age at menarche. The odds ratio was used to evaluate if the following variables are statistically significant risk factors for endometrial cancer: hypertension, diabetes, oral contraceptive pills, nulliparity, family history of cancer (breast, ovary, endometrium and colon), smoking, body mass index (BMI), over weight $>25 \mathrm{~kg}$. A multivariate analysis was performed to determine which of the risk factors is independent. For this the Binary Logistic Regression and Multiple logistic regression tests were done.

\section{Results}

Menstruation time is associated with high incidence of endometrial adenocarcinoma

The study was a case control study. Thirty postmenopausal, black patients were undergone transvaginal sonar showing endometrial thickness more than $4 \mathrm{~mm}$ and were also histologically proven with endometrial cancer. Other thirty postmenopausal black women who were clinically normal without any signs or symptoms of malignancy as their endometrial thickness were less than $4 \mathrm{~mm}$ and who were not on hormone replacement therapy were recruited. In the questionnaire we put emphasis on the relation of menarche and menopause age and length of menstruation time with the occurrence of endometrial cancer. The mean age at menarche in the study group was 13.80 years (range 11 to17 years) and in the control group was 14.83 years (range 12 to 20 years). The mean age at menopause in the study group was 53.86 years (range 47 to 60 years) and in the control group was 49.90 (range 45 to 60 years). The mean length of menstruation time in years in the study group was 40.20 years (range 31-49 years) and in the control group was 34.86 years ( range $27-46$ years). All these 
a.

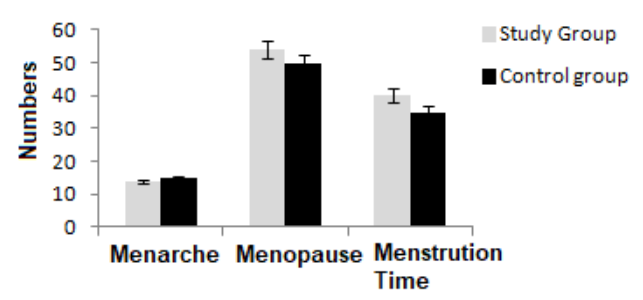

b.

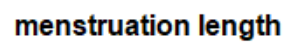

"Study Group $=$ Control group

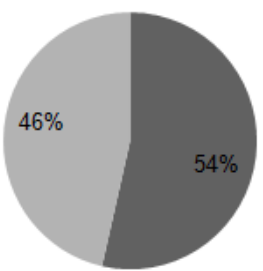

Figure 1. a) Bar Diagram Representation of Population of Postmenopausal Black Women Shows Mean + SD of early Menarche Age, Late Menopause Age and Menstruating Period from n=30 Control and Study Groups. b) Menstruation length among both the groups has been represented using percentage pie diagram.

parameters were significantly higher in the study group with $\mathrm{p}=0.044854848, \mathrm{p}=0.00049335$ and $\mathrm{p}=4.3745205$ for menarche, menopause and menstruating length respectively using the ANOVA test (Figure 1).

\section{Hypertension has positive impact on endometrial} adenocarcinoma incidence

On studying the relation between hypertension and occurrence of endometrial cancer we found 28 patients among 30 postmenopausal black women within study group. On the other hand in control group only 13 out of 30 subjects were found to be hypertensive. The calculated odds ratio between these two groups is 18.31 which are quite high. On analyzing this cohort groups level of hypertension were found to be significantly related to endometrial carcinoma among postmenopausal women (Figure 2a and 2b).

Serum oestradiol level regulates endometrial adenocarcinoma occurrence

In order to find the relation of blood oestradiol with classical adenocarcinoma, we observed serum level oestradiol in a group of 30 patients with 20 high grade, 6 intermediate grade and 4 low grade endometrial adenocarcinoma with mean age of 63 years 4 months.

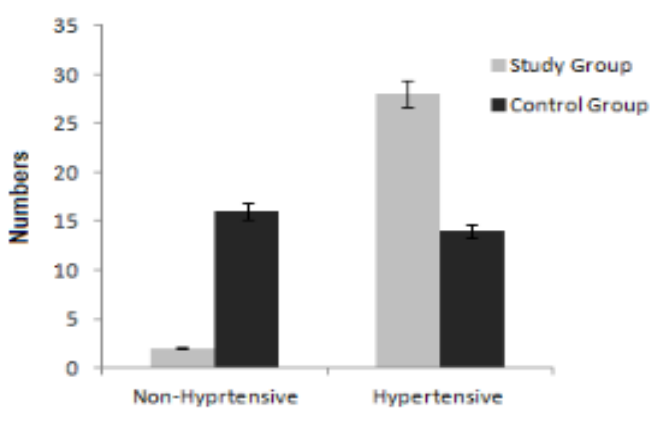

Figure 2. Bar Diagram Represents a Direct Relation between Hypertension and Endometrial Cancer among Women Population of South Africa.
A group of 30 normal subjects was kept as control with mean age of 61 years 8 months. All subjects were postmenopausal with no significant differences in their mean age, which was calculated using t-test. We observed blood oestradiol level in the study group to be 110.53 $\mathrm{pmol} / 1$ whereas control group shows $20.96 \mathrm{pmol} / 1$. Anova test analysis shows significantly higher level of oestradiol in the study group with $p=3.59835$. Among the study group 4 patients used oral contraceptive in comparison to 1 in the control group. The calculated odds ratios are 1.45 and 4.46 which are highly significant (Figure 3 ).

Diabetes, smoking habits, obesity etc have some influence on cancer incidence but statistically insignificant

The other factors like nulliparity, family history and obesity also play a vital role in endometrial cancer. Since nulliparity increases the chance of oestrogenic stimulation of the endometrium for longer period a slight rise in calculated odds ratio is vital. Here we found four patients in the study group were nulliparous compared with 1 in the control group. The calculated odds ratio was 4.46 which is highly significant. Four patients in the study group had a family history of cancer (endometrial/colon) compared with 2 in the control group. The calculated

\section{Oestradiol level}

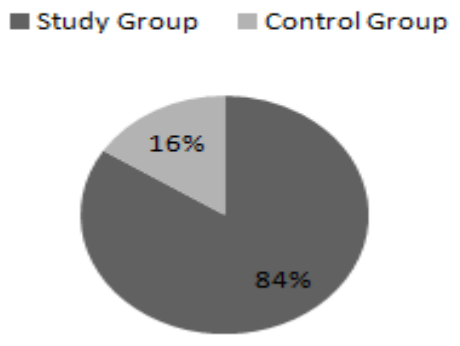

Figure 3. Bar Diagram Representing Observed Blood Oestradiol Level in the Study Group and in Control Group with an Average Mean Value of $110.53 \mathrm{pmol} / \mathrm{l}$ and $20.96 \mathrm{pmol} / \mathrm{l}$ Respectively, $\mathrm{n}=30$ each group. 
a.
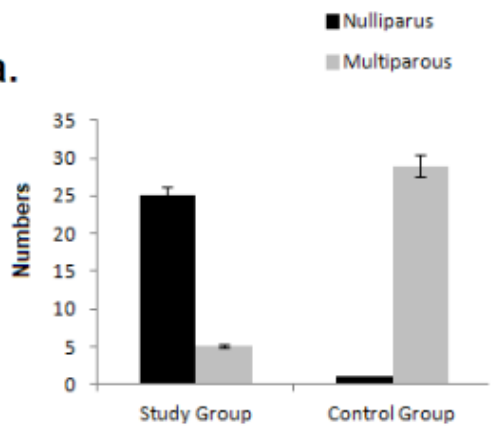

d.

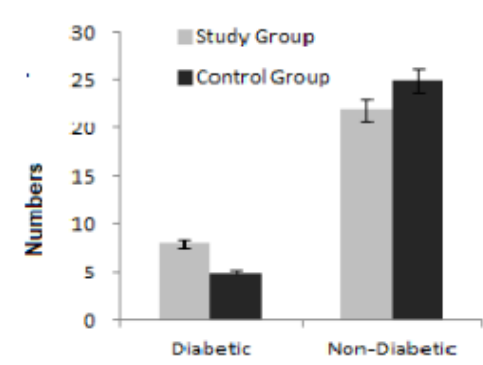

b.

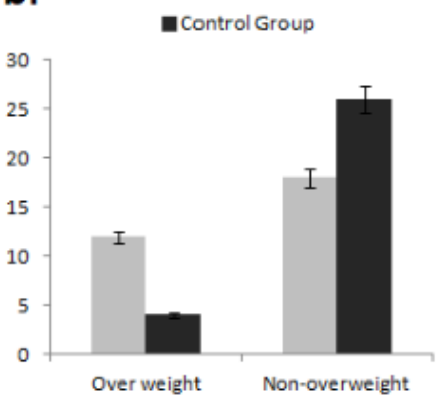

c.

\section{Family History}

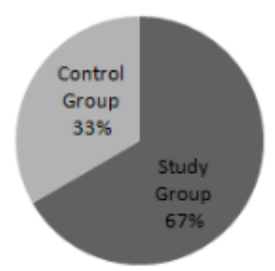

f.
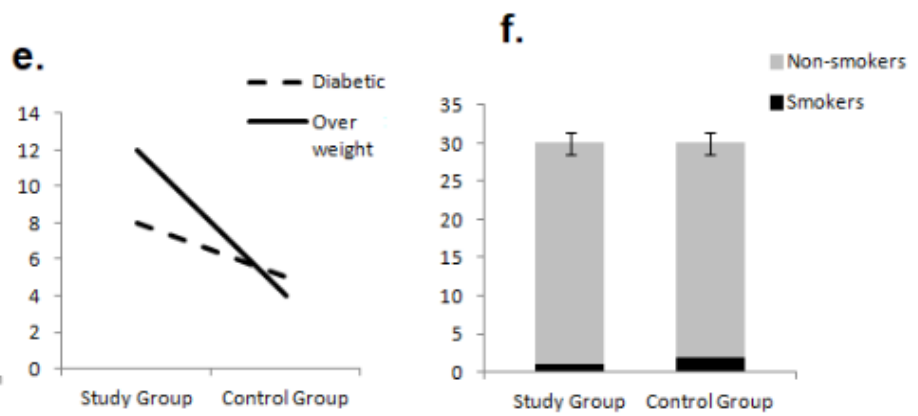

Figure 4. a) Nulliparity is more Common among Women with Endometrial Cancer than the Normal ones as Represented in the bar Diagram. b) Bar diagram shows over-weight population is even higher in endometrial cancer patients. c) Study group patients show a significant higher percentage of family history of endometrial cancer, represented by pie chart. d) Frequency of diabetic is more in the study group than in the normal. e) Statistical representation of relation between overweight and diabetes showing overweight results in diabetes which ultimately responsible for the occurrence of endometrial cancer. f) Statistical analysis shows no significant relation between endometrial cancer and habit of smoking.

odds ratio was 2.15 which is significantly high, $(95 \%$ confidence interval $0.4 ; 12.3$ ), (Figure $4 a$ ).

Obesity is considered to be one of the major factors related to endometrial cancer as fat tissue can change some other hormones into oestrogens, thus increasing oestrogen in circulating blood. Fourteen patients in the study group had a body mass index (BMI) of more than 30 compared with 6 in the control group. The odds ratio was 3.5 which is significant. A BMI of 30 was chosen as a cut-off as above it is obese and below is overweight. 12 patients in the study group were overweight by over 25 $\mathrm{kg}$ compared with 5 in the control group. The odds ratio was 3.3 (Figure 4b).

Four patients in the study group had a family history of cancer (endometrial/colon) compared with 2 in the control group. The calculated odds ratio was 2.15 which is significantly high (95\% confidence interval $0.4 ; 12.3)$, (Figure 4c).

Endometrial cancer is more common in women with diabetes, because diabetes is more prevalent in people who are overweight. 8 patients in the study were diabetic compared to 6 in the control group calculating its odds ratio to be significantly high of 4.46 (Figure $4 \mathrm{~d}$ and $4 \mathrm{e}$ ).

Among the various leading factors we checked smoking habits of each patient in normal and study groups. Only one patient in the study group had a history of smoking and 2 in the control group had the same. The odds ratio was 0.486 which was not significant thus further proving occurrence of endometrial cancer is not associated with the habit of smoking. This also further validated with the transvaginal sonar of both the groups showing no significant alterations on the walls of endometrium among normal patients with smoking habit (Figure 4f).

\section{Discussion}

The use of univariate analysis in this small cohort of patients and controls showed increased blood oestrogen level, late menopause, early menarche, and long menstruating time in years, nulliparity, and overweight by more than $25 \mathrm{~kg}$, hypertension, and diabetes, family history of the same or related colorectal carcinoma are found to have significant role in the development of carcinoma of the endometrium.

Our study agrees with that of earlier published data that there is a strong direct association of circulating estrogens, androgens and an inverse association of SHBG levels with endometrial cancer in postmenopausal women. The effect of elevated androstenedione and testosterone on disease risk seems to be mediated mainly through the conversion to oestrogens. Our study on association of diabetes with increased risk of endometrial cancer also supports the previous work of Anderson KE et al where the odds ratio of relative risk (RR) for postmenopausal women with diabetes versus women without diabetes was 1.43 [95\% confidence interval (CI), 0.98-2.1]. Similarly in our study we found diabetes to be a significant risk factor with an odds ratio 1.45 . 
Our study found higher significant relation between hypertension and endometrial cancer with an odds ratio 18.31 whereas the previous result (9) showed association of hypertension with endometrial cancer having odds ratio 2.4. but we found hypertension as significant risk factor with an odds ratio 18.31 .

In this study we observed nulliparity has a significant association with the risk of developing endometrial cancer with an odds ratio of 4.46. This data also confirms the earlier published literatures. This study also contradicts the previously reported data of inversely association of use of oral contraceptive hormone and endometrial cancer risk (OR, 0.36; 95\% CI, 0.14-0.90). A possible explanation is that the types of contraceptive pills used were oestrogen only preparations.

Smoking status was assessed in this cohort patients groups. Cox proportional hazards models were used to calculate multivariate relative risks (RR), controlling for endometrial cancer risk factors. Compared to non-smokers, the multivariate RR of endometrial cancer was significantly lower among both current smokers $(\mathrm{RR}=0.63 ; 95 \% \mathrm{CI}=0.50-0.79)$ and past smokers $(\mathrm{RR}=0.73 ; 95 \% \mathrm{CI}=0.62-0.87)$. The risk was lower among women who smoked 35 or more cigarettes a day $(\mathrm{RR}=0.60 ; 95 \% \mathrm{CI}=0.39-0.91)$ and among those who smoked for 40 or more years $(\mathrm{RR}=0.63 ; 95 \% \mathrm{CI}=0.45-0.87)$. These data indicate that both current and past smoking is associated with a lower risk of endometrial cancer. In this study, when smoking history was compared between the study and the control groups, the odds ratio was significantly lower than 1 . The protective effect of smoking on the incidence of endometrial cancer cannot be quantified from this small group of subjects.

In this study we even observed overweight by more than $25 \mathrm{~kg}$ to be statistically significant risk factor for endometrial cancer. The level of risk factor for endometrium carcinoma is related to the degree of obesity and is increased 10 fold for the women who are more than $23 \mathrm{~kg}$ overweight. BMI of more than 30 was found to be a risk factor in this study with an odds ratio of 3.5 .

In summary, using univariate analysis, only the use of oral contraceptive pills is not in accordance with literature review. All the other variables assessed agree with published literature. Using multivariate analysis this study identified high blood oestradiol level and hypertension as independent risk factors. This confirms the previous findings [10] who found that elevated blood oestradiol level is a significant risk factor and as reported earlier [11] linked hypertension with endometrial cancer.

Postmenopausal African women are at risk if they have high blood oestradiol level, as seen in this study. These women need to be screened further for endometrial cancer for which a prospective case control study will be needed.

This study requires further studies in genetics in the same population group using larger number of patients and compared with other population groups to determine if there exists a genetic predisposition in the African women population in the smallest province (geographically) of South Africa for having such a high incidence of endometrial cancer relative to the population. It also requires studying whether the independent prognostic factors (hypertension and high blood oestradiol level) are related with the stage, age, differentiation or other prognostic factors.

\section{References}

1. Wynder EL, Escher GC, Mantel N. An epidemiological investigation of cancer of the endometrium. Cancer. 1966;19(4):489-520.

2. Kurman RJ, Kaminski PF, Norris HJ. The behavior of endometrial hyperplasia. A long-term study of "untreated" hyperplasia in 170 patients. Cancer. 1985;56(2):403-12.

3. Smith DC, Prentice R, Thompson DJ, Herrmann WL. Association of exogenous estrogen and endometrial carcinoma. The New England journal of medicine. 1975;293(23):1164-7.

4. JP B. Major issues regarding estrogen replacement therapy in postmenopausal women. J Womens Health. 1994;3.

5. Lacey JV, Jr., Brinton LA, Lubin JH, Sherman ME, Schatzkin A, Schairer C. Endometrial carcinoma risks among menopausal estrogen plus progestin and unopposed estrogen users in a cohort of postmenopausal women. Cancer epidemiology, biomarkers \& prevention : a publication of the American Association for Cancer Research, cosponsored by the American Society of Preventive Oncology. 2005;14(7):1724-31.

6. Kurman RJ, Scully RE. Clear cell carcinoma of the endometrium: an analysis of 21 cases. Cancer. 1976;37(2):872-82.

7. Langer RD, Pierce JJ, O'Hanlan KA, Johnson SR, Espeland MA, Trabal JF, et al. Transvaginal ultrasonography compared with endometrial biopsy for the detection of endometrial disease. Postmenopausal Estrogen/Progestin Interventions Trial. The New England journal of medicine. 1997;337(25):1792-8.

8. Van den Bosch T, Vandendael A, Van Schoubroeck D, Wranz PA, Lombard CJ. Combining vaginal ultrasonography and office endometrial sampling in the diagnosis of endometrial disease in postmenopausal women. Obstetrics and gynecology. 1995;85(3):349-52.

9. Inoue M, Okayama A, Fujita M, Enomoto T, Tanizawa O, Ueshima H. A case-control study on risk factors for uterine endometrial cancer in Japan. Japanese journal of cancer research : Gann. 1994;85(4):346-50.

10. Lukanova A, Lundin E, Micheli A, Arslan A, Ferrari P, Rinaldi S, et al. Circulating levels of sex steroid hormones and risk of endometrial cancer in postmenopausal women. International journal of cancer. 2004;108(3):425-32.

11. Weiderpass E, Persson I, Adami HO, Magnusson C, Lindgren A, Baron JA. Body size in different periods of life, diabetes mellitus, hypertension, and risk of postmenopausal endometrial cancer (Sweden). Cancer causes \& control : CCC. 2000;11(2):185-92.

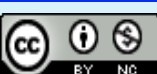

This work is licensed under a Creative Commons AttributionNon Commercial 4.0 International License. 